\title{
Integrated care for asthma: matching care to the patient
}

\author{
L.M. Osman*, M.I. Abdalla**, I.T. Russell**, J. Fiddes, \\ J.A.R. Friend*, J.S. Legge*, J.G. Douglas*
}

Integrated care for asthma: matching care to the patient. L.M. Osman, M.I. Abdalla, I.T. Russell, J. Fiddes, J.A.R. Friend, J.S. Legge, J.G. Douglas. @ERS Journals Ltd 1996.

ABSTRACT: The purpose of the present study was to investigate whether criteria associated with assignment of asthma patients between general practice (GP) care alone, integrated care (shared between GP care and hospital clinic) or conventional specialist review could be identified, and whether outcomes for these patients differed over the next 12 months.

Seven hundred and sixty four patients with a diagnosis of asthma and previously assigned to either integrated care or clinic care were reviewed after 1 year and reassigned. These patients were then followed for another $\mathbf{1 2}$ months and clinical data were collected over this time.

After 12 months in clinic care or integrated care, assignment to integrated care was predicted by previous participation in integrated care (OR 2.94), patient preference for integrated care (OR 3.7), no admission (OR 1.56), fewer steroid courses during the previous year $(\mathrm{OR} 0.88)$ and nonattendance at review $(O R$ 0.43) in the previous 12 months. Patient discharge to GP care was predicted by higher level of forced expiratory volume in one second (FEV1) (OR 1.49), lower number of GP consultations for troublesome asthma (OR 0.78), and nonattendance for review in the preceding year (OR 2.15). In the following 12 months, the three groups of patients differed significantly in hospital admissions (Discharged= 0.008; Integrated $=0.12 ;$ Clinic $=0.31)$, bronchodilators prescribed $($ Discharged $=8.5$; Integrated=10.2; Clinic=13.9), GP consultations (Discharged=1.3; Integrated=3.0; Clinic $=4.1$ ) and oral steroid courses (Discharged=0.62; Integrated=1.7; Clinic=2.4).

Patients assigned to integrated care, clinic care or discharged to general practice care form three distinct patient populations differing retrospectively and prospectively in morbidity and admission risk. In particular, patients assigned to integrated care fall midway in risk and morbidity between those discharged or those retained in clinic care. These results suggest that integrated care provides general practitioners with a system of management for asthma patients, for whom they do not wish frequent specialist review but who they do not believe can safely be discharged to general practice care only.

Eur Respir J., 1996, 9, 444-448.

\author{
*Thoracic Medicine Unit, City Hospital, \\ Aberdeen, Scotland, UK. \\ **Health Services Research Unit, University \\ of Aberdeen, Foresterhill, Scotland, UK. \\ Correspondence: L.M. Osman \\ Chest Clinic \\ City Hospital \\ Aberdeen \\ Scotland AB9 8AU \\ UK \\ Keywords: Asthma \\ integrated care
}

Received: March 231995

Accepted after revision October 71995
In medical systems which have two tiered patient care, (specialist out-patient clinics and general practice (GP) care) it is believed that specialist care allows greater costeffectiveness and the focusing of clinical expertise. However, it can be associated with gaps in communication between the specialist who sees the patient at regular intervals in an out-patient clinic and the GP, who sees the patient irregularly and at times of crisis with little knowledge of what is being provided in the specialist clinic. Shared care is intended to address these problems of communication and to offer patients care in general practice which has the support of specialist expertise. Shared care has been defined as "the joint participation of hospital consultants and general practitioners in the planned delivery of care for patients with a chronic condition ... (involving) enhanced exchange of information over and above routine discharge and referral letters" [1].
In the most common form of shared care for chronic conditions, GPs have day-to-day clinical responsibility for the care of the patient including review care and critical episode care, with an annual review for the patient at a specialist out-patient clinic. At GP reviews, a standard record sheet is usually completed by the GP and is returned to the hospital. From the hospital updated patient summaries are returned to the GP including any comments or advice on treatment from the patient's consultant. Patients are entered into shared care schemes after they have had specialist referral and assessment. Shared care enables more patients to receive specialist advice and better exchange of information between GP and specialist. Integrated care schemes have been introduced in several areas of out-patient care for chronic conditions, including hypertension [2, 3] and diabetes [3, 4], but few shared care schemes in asthma have been reported [1]. 
An integrated care scheme for asthma was implemented and evaluated in a large scale clinical trial (Grampian Asthma Study of Integrated Care (GRASSIC)) [5] in the North East of Scotland. All practices in Grampian took part in the study. In this trial, patients were randomly assigned between integrated care and conventional clinic care. Patients in the integrated care arm of the study were reviewed by their GP every 3 months, with review data being returned to the clinic to be seen by the referred clinician. At any time, patients could be referred back into full clinic care if the GP felt it appropriate. At the end of 12 months, all patients were reviewed at the chest clinic.

This randomized trial found no difference between the integrated and conventional specialist review groups in clinical outcomes (admissions, lung function, morbidity measures and acute episodes) or quality of life outcomes, but patients in integrated care were more likely to feel "in control" of their asthma. Both GPs and patients were in favour of this form of shared care scheme [5, 6].

All of the 89 practices in Grampian (312 principals) took part in the GRASSIC study, and after the GRASSIC evaluation integrated care was continued as a clinical option for all asthma patients referred to specialist care in Grampian. At the end of the GRASSIC trial patients who had taken part were reviewed and their GPs asked whether they now wished their patient's care to be undertaken in general practice alone ("discharged to general practice care"), or in the integrated care scheme, or in conventional hospital specialist review.

In the current management of the Grampian Integrated Care scheme the decision to enter a patient into the scheme is made after an initial assessment covering not less than three clinic attendances. Patients with asthma are referred from throughout Grampian, thus integrated care serves urban and rural and large and small practices. Most GPs have one to two patients in integrated care. No single GP has more than eight integrated care patients.

It has been suggested that GPs are reluctant to recommend that patients be discharged from specialist review, even when their asthma is well-controlled and stable [7]. If this were the case in our scheme, integrated care might be recommended by GPs for patients who could equally well have been discharged. We wished to investigate whether distinct retrospective criteria were associated with differing assignment, and whether patients recommended for integrated care would, in a follow-up year, differ from patients discharged to general practice care alone, or retained in specialist review care.

\section{Method}

\section{Research design}

Seven hundred and sixty four patients attending chest clinics in Aberdeen, Banff, Elgin and Peterhead were reviewed and reassigned at the end of the GRASSIC randomized trial year. This was $95 \%$ of the original 801 patients in the trial. Thirteen patients had died during the GRASSIC trial year and the remaining 24 had been lost to the study. Patients were aged 16 yrs or over, with pulmonary function reversibility of at least $20 \%$, a diagnosis of asthma confirmed by a chest physician and consistent with the International Guidelines criteria for the diagnosis of asthma [8,9]. All patients were at Step 2 or above of the Chronic Management Guidelines (BTS) (moderate to severe asthma). At the beginning of the follow-up year average peak expiratory flow (PEF) of patients taken at clinic review was $68 \%$ predicted (SD $27 \%$ ) and forced expiratory volume in one second (FEV1) $75 \%$ predicted (SD 27\%).

All but 85 of these patients had been randomly assigned during GRASSIC between conventional clinic and integrated care. During GRASSIC, these 85 had been retained in clinic care because they were judged too severe for randomization but full data had been collected for them. In the following analyses, this group is identified as "not randomized in GRASSIC year".

Data on all clinical outcomes for patients over the GRASSIC year were provided by the GPs of the patients (prescriptions for inhaled steroids and bronchodilators oral steroid courses, and number of GP consultations for troublesome asthma over the GRASSIC year). PEF was measured at the final GRASSIC review. At the end of the GRASSIC trial, GPs were sent a questionnaire for each patient, which asked whether they wished the patient to be discharged to GP care or assigned to integrated or clinic care. This completed questionnaire was used by the clinician at the review deciding assignment for the next 12 months. All patients had been interviewed at home at the end of the GRASSIC evaluation and during this interview had been asked their preference for continuing care. Clinicians were blind to the answers given in this interview.

Twelve months later, GPs were asked about clinical events for all patients over this period, including those discharged to GP care alone. These clinical events matched those collected during the GRASSIC trial. Data were provided by each GP on number of prescriptions for inhaled steroids and bronchodilators, oral steroid courses, $\mathrm{PEF}$ at the end of the follow-up year, and number of GP consultations for troublesome asthma over the year. Nine $(10 \%)$ practices in Grampian do not have computerized prescribing systems and these practices provided data by review of case notes. Hospital admissions were obtained from hospital records. Clinical outcomes over the follow-up year were compared between the discharged, integrated care and clinic care groups.

\section{Statistical analysis}

Logistic regression was used to identify which clinical measures and patient characteristics in the GRASSIC trial year before assignment best predicted assignment between care types at review. Clinical measures considered were PEF and FEV1 at review, number of GP consultations for asthma episodes, oral steroid courses and hospital admissions during the GRASSIC trial. Patient 
Table 1. - Care type assigned at review (end of GRASSIC year) by care type during GRASSIC year

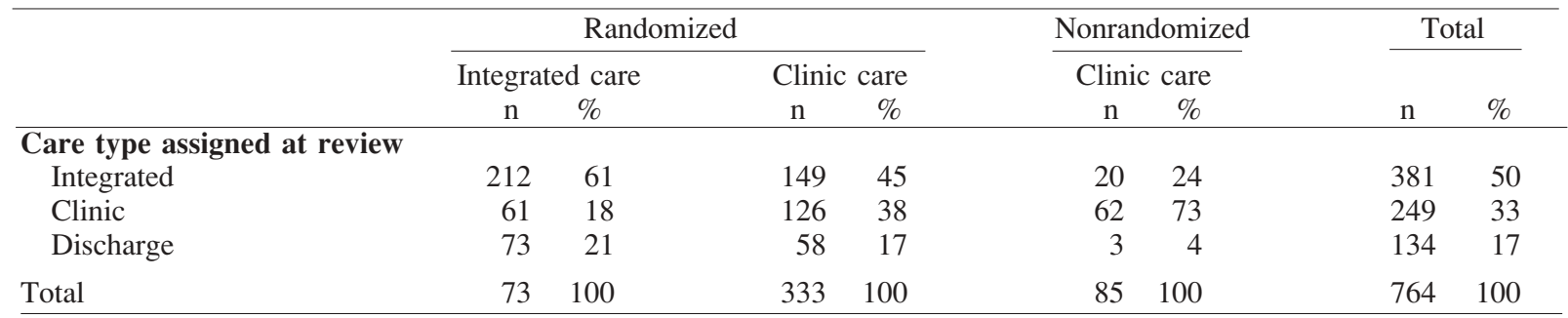

GRASSIC: Grampian Asthma Study of Integrated Care.

characteristics were age, sex, whether during the previous GRASSIC study the patient had been randomized between integrated and clinic care, patient preference for care (assessed by questionnaire at the end of the study) and whether the patient was one of the 86 patients (44 $(11 \%)$ of clinic care patients, and $42(12 \%)$ of integrated care patients) who had not attended any review consultations or GP reviews. These 86 patients were identified as "nonattenders" in the analysis of factors predicting care type assignment. A first analysis was carried out to identify predictors for discharge to GP care. Among nondischarged patients, a second analysis identified predictors of allocation to integrated care rather than conventional clinic care.

Analysis was stepwise. It finished when none of the remaining variables improved the maximum log likelihood by an amount significant at the $5 \%$ level.

Means and $95 \%$ confidence intervals $(95 \% \mathrm{CI})$ for the clinical outcomes of the follow-up year were calculated using the GLIM package [10]. A Poisson error structure and a log link function were used as these measurements were not normally distributed.

\section{Results}

Mean (SD) age of patients was 50 (17) yrs. Forty three percent were male. Average length of time since asthma was diagnosed was 18 (17) yrs.

Table 1 shows review decision at the end of the GRASSIC year. One hundred and thirty four patients were discharged to GP care, 381 were retained or transferred to integrated care, 249 were returned or transferred to clinic care. Ninety two percent of GP recommendations for clinic care were followed for patient assignment, $76 \%$ of GP recommendations for integrated care, and $74 \%$ of recommendations for discharge. Patients who had been randomized to integrated care during the GRASSIC trial were more likely to continue in integrated care in the follow-up year than patients who had been randomized to clinic care during GRASSIC. Likelihood of discharge appeared to be similar for either arm of the GRASSIC trial.

A logistic regression analysis was carried out in order to identify the significant independent predictors of discharge to GP care at the review (table 2). Discharge was significantly related to higher FEV1 at the GRASSIC review, fewer GP consultations for troublesome asthma during the GRASSIC trial year, and asthma not severe enough to prevent randomization in the trial year. The small number of patients who did not attend reviews ("nonattenders") were more likely to be discharged. Having no hospital admission during the previous year fell just short of significance in predicting discharge from specialist care. Patients in integrated care during GRASSIC were not more likely to be discharged to GP care than patients randomized to conventional clinic care.

Table 3 lists six significant predictors of assignment to integrated care rather than clinic care. The odds of assignment to integrated care were three times higher for patients in integrated care during the trial year. Patients who in their final GRASSIC interview had stated a preference for integrated care were almost four times more likely to be assigned to it. No admission in the trial year increased the odds of assignment to integrated care by $56 \%$. Patients who had been kept in clinic care in the previous year (judged not eligible for randomization between integrated and clinic care) were $67 \%$ less likely

Table 2. - Predictors of discharge to general practice care at final GRASSIC review $(n=764)$

\begin{tabular}{|c|c|c|c|c|}
\hline & $\begin{array}{c}\text { Discharged } \\
(\mathrm{n}=134)\end{array}$ & $\begin{array}{l}\text { Assigned to integrated } \\
\text { or clinic care at review } \\
(n=630)\end{array}$ & $\begin{array}{c}\text { OR for } \\
\text { discharge\# }\end{array}$ & $95 \% \mathrm{CI}$ \\
\hline FEV1 at final GRASSIC review \% pred $\# \# *$ & $86.7(81.7-91.7)$ & $72.1 \quad(70.1-74.1)$ & 1.49 & $1.20-1.86$ \\
\hline Number of GP consultations ${ }^{\# \#}$ & $1.3(0.8-1.8)$ & $3.3(3.0-3.6)$ & 0.78 & $0.69-0.82$ \\
\hline Not randomized during GRASSIC year ${ }^{\dagger}$ & $3 \quad(2)$ & $82 \quad(13)$ & 0.20 & $0.06-0.66$ \\
\hline "Nonattender" during GRASSIC year ${ }^{\dagger}$ & (18) & $(10)$ & 2.15 & $1.21-3.81$ \\
\hline No admissions during GRASSIC year**† & (97) & $545 \quad(87)$ & 0.38 & $0.13-1.06$ \\
\hline
\end{tabular}

\#: as estimated from stepwise logistic regression, in order of stepwise selection; \#\#: mean, and 95\% CI in parenthesis; ${ }^{\dagger}$ : absolute value, and percentage in parenthesis; *: the effect of $10 \%$ change in this variable; **: significant only at $7 \%$ level. GRASSIC: Grampian Asthma Study of Integrated Care; OR: odds ratio; 95\% CI: 95\% confidence interval; FEV1: forced expiratory volume in one second; GP: general practitioner; $\%$ pred: percentage of predicted value. 
Table 3. - Predictors of assignment to integrated care among patients not discharged to general practice care at end of GRASSIC $(n=630)$

\begin{tabular}{|c|c|c|c|c|}
\hline & \multicolumn{2}{|c|}{ Assignment at review: } & \multirow{2}{*}{$\begin{array}{l}\text { OR for assignment } \\
\text { to integrated } \\
\text { care at review }\end{array}$} & \multirow[t]{2}{*}{$95 \% \mathrm{CI}$} \\
\hline & $\begin{array}{l}\text { Integrated care } \\
\quad(\mathrm{n}=381)\end{array}$ & $\begin{array}{l}\text { Clinic care } \\
(\mathrm{n}=249)\end{array}$ & & \\
\hline $\begin{array}{l}\text { Randomized to integrated care during } \\
\text { GRASSIC year*† }\end{array}$ & $212(56)$ & $61(24)$ & 2.94 & $(1.95-4.36)$ \\
\hline $\begin{array}{l}\text { Patient preferred integrated care at end of } \\
\text { GRASSIC year }{ }^{\dagger}\end{array}$ & $279(73)$ & 104 (42) & 3.7 & $(2.5-5.4)$ \\
\hline No admission during GRASSIC year ${ }^{\dagger}$ & $347(91)$ & $198(80)$ & 1.56 & $(1.05-2.3)$ \\
\hline Not randomized during GRASSIC year ${ }^{\dagger}$ & $20(5)$ & $62(25)$ & 0.33 & $(0.19-0.62)$ \\
\hline "Nonattender" during GRASSIC year & $26(7)$ & $36(14)$ & 0.43 & $(0.23-0.80)$ \\
\hline Number of oral steroid courses\#\# & $1.7(1.5-1.9)$ & $2.6(2.3-3.0)$ & 0.88 & $(0.81-0.96)$ \\
\hline
\end{tabular}

\#: as estimated from stepwise logistic regression, in order of stepwise selection; *: among patients randomized during GRASSIC trial; \#\#: mean, and 95\% CI in parenthesis; ${ }^{\dagger}$ : absolute value, and percentage in parenthesis. For definitions see legend to table 2.

Table 4. - Clinical events during follow-up year

\begin{tabular}{|c|c|c|c|c|c|c|c|}
\hline \multirow{3}{*}{$\frac{\text { Clinical events }}{\text { Hospital admissions }}$} & \multicolumn{7}{|c|}{ Mean $(95 \%$ CI $)$} \\
\hline & \multirow{2}{*}{$\frac{\mathrm{n}}{739}$} & \multicolumn{2}{|c|}{ Discharged } & \multicolumn{2}{|c|}{ Integrated care } & \multicolumn{2}{|c|}{ Clinical care } \\
\hline & & 0.008 & $(0.001-0.05)$ & 0.12 & $(0.09-0.17)$ & 0.31 & $(0.25-0.39)$ \\
\hline Bronchodilator prescribed & 550 & 8.5 & $(7.1-10.1)$ & 10.2 & $(9.3-11.3)$ & 13.9 & $(12.5-15.4)$ \\
\hline Inhaled steroids prescribed & 552 & 5.6 & $(4.6-6.6)$ & 6.3 & $(5.8-6.9)$ & 7.2 & $(6.5-7.9)$ \\
\hline Oral steroid courses & 579 & 0.6 & $(0.4-0.9)$ & 1.7 & $(1.5-1.9)$ & 2.4 & $(2.1-2.8)$ \\
\hline GP consultations & 601 & 1.3 & $(1.0-1.8)$ & 3.0 & $(2.7-3.4)$ & 4.1 & $(3.6-4.6)$ \\
\hline $\begin{array}{l}\text { PEF at end of } \\
\text { follow-up year \% pred }\end{array}$ & 508 & 71 & $(68-74)$ & 67 & $(65-69)$ & 63 & $(61-66)$ \\
\hline
\end{tabular}

PEF: peak expiratory flow. For further definitions see legend to table 2.

to be assigned to integrated care at review. Patients who did not attend reviews had their odds of assignment to integrated care reduced by $57 \%$. Each additional steroid course used by a patient decreased their probability of assignment to integrated care by $12 \%$.

Details of the follow-up year were returned for 607 (79\%) patients. Table 4 shows clinical events over the following 12 months as reported by the patients' GPs. On all of these measures the three groups form distinctly separate populations. Only one patient in the discharged group was admitted during the follow-up year. After 12 months, patients discharged to general practice care had the highest average PEF, had made the least GP consultations for troublesome asthma, had used fewer oral steroid courses, and had had fewer prescriptions for bronchodilators. Patients assigned to integrated care fell midway between those discharged and those assigned to clinic care. Their admission rate was 0.12 compared to 0.31 in the clinic care group, they used 1.7 oral steroid courses compared to 2.4 in the clinic group, and their mean PEF was 346 compared to 319 in the clinic group.

At the end of the follow-up year, 36 patients were discharged from integrated care and 18 were returned to clinic care. Ten patients died in integrated care (two as a result of asthma) and eight in clinic care (four as a result of asthma).

\section{Discussion}

The previous GRASSIC study of integrated care [5] showed that there were no differences in clinical outcome for patients with asthma randomized between integrated and conventional clinic care. In clinical practice, patients are assigned to care on the basis of medical decision and will not be randomly allotted to different care types. The present study showed that patients assigned by GPs and specialists to different care types differ retrospectively and prospectively in clinical characteristics and outcomes.

Discharge from specialist care was positively related to FEV1, negatively related to GP consultations for troublesome asthma during the previous year, and more likely for patients who had not attended any reviews. Lack of contact for acute episodes and nonattendance at review suggests that these patients have been stable, with wellcontrolled asthma, and that both GPs and consultants judge this group, $17 \%$ of the total patient group, to be appropriately managed without specialist review.

For the remaining 630 patients, the decision to be made was whether they would be best assigned to integrated care or conventional clinic care. Each oral steroid course prescribed during the GRASSIC trial year decreased the odds of assignment to integrated care in the follow-up year by $12 \%$. Having had no hospital admission increased the odds of assignment to integrated care by $56 \%$. Having been randomized to integrated care during the trial markedly increased the likelihood of review assignment to integrated care for the follow-up year.

Patient preference for integrated care also increased the likelihood that the patient would be assigned to integrated care. This suggests that patient and GP confidence is developed by experience of integrated care $[5,6]$. 
For one reason or another, there will be patients who do not comply with the review requirements of integrated care. In this study, there were 82 "nonattenders". These patients were less likely to be assigned to integrated care. Most of them were returned to conventional clinic care. Twenty nine percent of the "nonattenders" were discharged. It seems likely that GPs are prepared to recommend that stable patients who do not attend reviews should be discharged from specialist care, but that they prefer that the less well-controlled nonattender be in conventional clinic care rather than integrated care.

It has been suggested that GPs are reluctant to recommend that patients be discharged from specialist care, even when these patients have stable and well-controlled asthma. In one study [7], of 164 clinic patients identified as stable, GPs believed $27 \%$ were suitable for discharge to general practice care alone. In the present study, $17 \%$ of patients were discharged following GP recommendation and consultant review. There was a high level of agreement between consultants and GPs, and patients not discharged differed significantly both retrospectively and prospectively in clinical measures. The findings of the follow-up year in this study support the validity of the GP and consultant decision not to discharge. Patients assigned to integrated care experienced significantly worse outcomes (more hospital admissions and GP consultations, higher consumption of asthma medication, and worse pulmonary function) than patients discharged at review.

However, patients assigned to integrated care experienced much better outcomes than patients assigned to conventional clinic care. We have previously shown that there are no differences in outcome for patients randomly assigned between integrated and clinic care. It is unlikely, therefore, that the differences found in the present study are due to the differences in care now producing such different outcomes. Similarly since $90 \%$ of our practices have computerized systems for retrieval of prescribing data any bias in recording across the 3 groups should be minimal. We suggest that the differences are due to the ability of GPs and consultants to identify levels of risk for patients. If true, this explanation confirms the case for an integrated care scheme that meets the needs of patients not so stable as to merit discharge from consultant care, but not so severe as to need frequent outpatient consultations.

During the GRASSIC study, 90\% of patients referred to the Chest Clinic were randomized between conventional and clinic care and no disadvantages were found for those in integrated care. This strongly suggests that after an initial assessment period almost all patients referred to specialist care can be entered into any similar integrated care system. The present study shows that after 12 months in an integrated care scheme consultants and GPs can identify patients suitable for discharge from those who should continue in integrated care (and those who should return to clinic care). The study suggests that integrated care fills a need for an appropriate scheme of management for asthma patients who do not require frequent specialist clinic review but cannot safely be discharged.

These results also suggest that it may not be necessary or cost-effective as standard practice to provide all care for chronic conditions, such as asthma, within a specialist care system. In countries with a developed primary care system such as the UK and the Netherlands the general practitioner will be able to manage the majority of asthma patients without continuing specialist involvement.

Acknowledgements: The authors thank the general practitioners and practice nurses of Grampian for their continuing co-operation and D. Peat for his work in entering the data for this study. The Senior Research Fellowship of LMO is funded by the National Asthma Campaign, UK.

\section{References}

1. Hickman M, Drummond N, Grimshaw J. The operation of shared care for chronic disease. Health Bulletin 1994; 52: 118-126.

2. Petrie JC, Robb OJ, Webster J, Scott JK, Jeffers TA, Park MD. Computer assisted shared care in hypertension. Br Med J 1985; 290: 1960-1963.

3. Petrie JC, Webster J, Jeffers TA, Bell DMR. Computerassisted shared care: the Aberdeen Blood Pressure Clinic. $J$ of Hypertension 1989; 7: 103-108.

4. Day JL, Humphreys H, Alban-Davies H. Problems of comprehensive shared diabetes care. Br Med J 1987; 294: $1590-1592$.

5. GRASSIC. Integrated care for asthma: a clinical social and economic evaluation. Br Med J 1994; 308: 559-564.

6. Van Damme RAE, Drummond NA, Beattie JAG, Douglas JG. Integrated care for patients with asthma: views of general practitioners. Br J Gen Pract 1994; 44: 9-13.

7. Leitch AG, Parker S, Currie A, King T, McHardy GJR. Evaluation of the need for follow up in an out-patient clinic. Respir Med 1990; 84: 119-122.

8. Sheffer A. International Consensus Report on diagnosis and treatment of asthma. Eur Respir J 1992; 5: 605-641.

9. Fabbri LM, Cogo P, Cosma P, Guidobono A, Ciaccia A. Diagnosis in adults. In: O'Byrne $\mathrm{P}$, Thomson NC, eds. Manual of Asthma Management. London, W.B. Saunders, 1995; pp. 83-102.

10. GLIM Working Party. The GLIM system release 3.77. Oxford, Numerical Algorithms Group, 1987. 